\title{
POLYMERIC TRIBOELECTRIC NANOGENERATOR: EFFECTS OF POLYMER TYPE, GEOMETRY, AND POROSITY ON TRIBOELECTRIFICATION
}

\author{
Hossein Abdoli and Siu N. Leung \\ Department of Mechanical Engineering \\ York University \\ Toronto, ON, Canada \\ sunny.leung@lassonde.yorku.ca
}

\begin{abstract}
Triboelectric nanogenerator (TENGs), based on the principles of triboelectrification and electrostatic induction, have recently been viewed as a promising approach to harvest mechanical energy, which would otherwise be wasted through dissipation to surrounding. Large contact area and large surface area have been identified as the basic design principles to promote the performances of TENGs. In this study, particulate leaching method was used to introduce and tailor the open-cell morphology of negative triboelectric layers in TENGs. The effects of material type, material geometry, open-cell morphology on the level of triboelectrification induced by mechanical motions were also investigated. Experimental results revealed that polyvinylidene fluoride (PVDF) performed better than high density polyethylene (HDPE) as the negative triboelectric layer of the TENG. Furthermore, increasing the contact area, reducing the thickness, and introducing fine pores would enhance the performances of TENGs.
\end{abstract}

Keywords-energy harvesting; geometry; open-cell morphology; polymer; triboelectricification

\section{INTRODUCTION}

Triboelectrification, in conjunction with electrostatic induction, has been developed into an innovative energy harvesting technology - triboelectric nanogenerators (TENGs) [1]. Although different types of technologies based on electrostatic, electromagnetism, and piezoelectricity exist to convert mechanical energy into electrical energy [2], TENGs have recently been drawing attentions. It is considered as a promising solution to harvest mechanical energy, which will otherwise be wasted, into useful energy. In addition, TENGs are also capable of powering sensors, portable electrics, and biomedical microsystems [1,3].

There are several different modes of TENGs. Among them, the contact-separation mode is one of the simplest ones. In this mode, two friction materials with different triboelectric properties contact and separate periodically. Each of them is attached to an electrode. During contact, electrons transfer from one material to the other because of their abilities to attract electrons. As a result, the material loses electrons would become positively charged while the other gains electrons would be negatively charged. Subsequently, the two oppositely charged materials separate and a potential difference will be generated between them. This will electrostatically induce a current flow along an external circuit connected to the two electrodes adhered by the two materials in order to compensate the potential difference. When the two materials are pressed again, the potential difference will decrease and a reverse current flow will be induced. This periodic action will in turn generate an alternating current [3].

Although triboelectrification is a well-known phenomenon, its fundamental is not fully understood yet. Various parameters can affect the degree of triboelectrification of materials, and thereby the performances of TENGs. These factors include humidity, external force applied to induce the periodic contact and separation of friction materials, chemical composition of the friction layers, geometrical parameters (e.g., thickness and contact area), and morphological parameters (e.g., shape and surface area).

Recently, researchers have conducted studies to investigate the effects of these governing factors on the degree of triboelectrification in order to optimize the performances of TENGs [1-3]. Wang revealed that microstructuring the surface of PDMS, which was used as the negative side of the friction materials, could influence the charge density accumulated on the material surfaces [4]. It is believed that the creation of pyramidbased micro-patterns is effective to enhance the contact area and potentially the triboelectrification. Lee et al. suggested that the introduction of a film-covered pillar structure on PDMS would enhance the conformable contact and adhesion energy, which would in turn increase triboelectrication [5]. Another recent work fabricated a hydrophobic polydimethylsiloxane (PDMS) sponge to study the effect of pore size and humidity on TENG parameters [2]. They observed that TENG voltage and current density greatly increased when PDMS pore size reduced from $10 \mu \mathrm{m}$ to $0.5 \mu \mathrm{m}$. Furthermore, the developed voltage only slightly decreased when relative humidity increased from 20 to $80 \%$, which showed reasonable stability of hydrophobic structure under different humidity conditions. Chen et al. posed that matrix of high dielectric nanoparticles like strontium titanate $\left(\mathrm{SrTiO}_{3}\right)$ in PDMS can enhance charge density of TENG and improve the power output of the device by five times [6]. 
This paper studied the effects of material type and material geometry (e.g., thickness and contact area) on the electrification of polymer-based TENGs. After identifying the appropriate material type, particulate leaching was used to introduce and tailor the open-cell structures of negative triboelectric layers. Parametric studies were conduct to elucidate how the open-cell morphology of the triboelectric layer influenced the output performances of TENGs. This work enhanced the understanding of material design on their triboelectriciation and provided new and effective strategy to enhance the performances of TENGs.

\section{EXPERIMENTAL}

\section{A. Materials}

Polyvinylidene fluoride (PVDF, Kynar 741, Arkema) and high density polyethylene (HDPE, Sclair ${ }^{\circledR}$ 2710, NOVA Chemicals) were used to fabricate the negative triboelectric layers of TENGs. Thermoplastic polyurethane (TPU, Estane ${ }^{\circledR}$ 2103-70A, Lubrizol) was used to fabricate the positive triboelectric layers of TENGs. Table I summarizes the physical properties of PVDF, HDPE, and TPU. Sodium chloride $(\mathrm{NaCl})$ was used as the leaching agent to fabricate porous negative triboelectric PVDF layers. The electrodes of TENGs were made by copper tapes with conductive adhesive. All materials were used as received without any modification.

TABLE I. PHYSICAL PROPERTIES OF PVDF, HDPE, AND TPU

\begin{tabular}{|l|c|c|}
\hline \multirow{2}{*}{ Polymer } & \multicolumn{2}{|c|}{ Physical Properties } \\
\cline { 2 - 3 } & Density & Young's Modulus \\
\hline HDPE & $951 \mathrm{~kg} / \mathrm{m}^{3}$ & $1000 \mathrm{MPa}$ \\
\hline PVDF & $1780 \mathrm{~kg} / \mathrm{m}^{3}$ & $1700 \mathrm{MPa}$ \\
\hline TPU & $1060 \mathrm{~kg} / \mathrm{m}^{3}$ & $2.1-5.2 \mathrm{MPa}$ \\
\hline
\end{tabular}

\section{B. Sample Preparation}

Solid triboelectric negative layers were fabricated by compression molding (Craver Press, $4386 \mathrm{CH}$ ) PVDF powders or HDPE pellets at $185^{\circ} \mathrm{C}$ and $140^{\circ} \mathrm{C}$, respectively, into samples with various thicknesses and areas. In the first step, the heat platens of the compression molding machine were brought into contact with the mold, which loaded with polymer powders or pellets, for 5 minutes to ensure the complete melting of the materials. Subsequently, the molding pressure was ramped up to $27 \mathrm{MPa}$ and was held at the set pressure for 5 minutes to fabricate the disc samples. Similar procedures were used to fabricate the solid TPU triboelectric positive layers, which were $0.5 \mathrm{~mm}$ thick.

Porous PVDF triboelectric negative layers were fabricated by compression molding and particulate leaching process using the procedures indicated below:

STEP 1. $\mathrm{NaCl}$ powders were sieved into different sizes (i.e., $<53$ $\mu \mathrm{m}$ and $53-250 \mu \mathrm{m}$,).

STEP 2. PVDF powders were dry-blended with $80 \mathrm{wt} \% \%$ sieved $\mathrm{NaCl}$ powders.

STEP 3. PVDF-NaCl mixtures were molded by a compression molding machine at $185^{\circ} \mathrm{C}$ and $27 \mathrm{MPa}$ into disc samples of desired thicknesses and areas. All porous samples are $0.50 \mathrm{~mm}$ in their thicknesses and $3.14 \mathrm{~cm}^{2}$ in their areas.

STEP 4. Molded samples were submerged under water at room temperature (i.e., $23^{\circ} \mathrm{C}$ ) for 72 hours to leach out the $\mathrm{NaCl}$ crystals. Water was changed regularly to avoid the saturation of $\mathrm{NaCl}$.

STEP 5. The open-cell PVDF samples were then dried in an oven at $50^{\circ} \mathrm{C}$ for 24 hours.

Table II summarizes the key parameters being studied in this work to elucidate the effects of material type, material geometry, and open-cell morphology of the negative triboelectric layer on the triboelectrification of the TENG.

TABLE II. PhysicAl PROPERTIES OF PVDF, HDPE, AND TPU

\begin{tabular}{|l|c|c|}
\hline \multicolumn{1}{|c|}{ Parameter } & Value & Unit \\
\hline Material Type & PVDF and HDPE & - \\
\hline Thickness & $0.15,0.22$, and 0.50 & $\mathrm{~mm}$ \\
\hline Area & $2.0,4.0,6.0$, and 8.0 & $\mathrm{~cm}^{2}$ \\
\hline Pore Size & $<53$ and $53-250$ & $\mu \mathrm{m}$ \\
\hline
\end{tabular}

\section{Sample Characterization}

The morphology and structure of the samples were characterized by a scanning electron microscope (SEM) (FEI Company Quanta 3D FEG. The cross-sections of porous PVDF samples were exposed by cryo-fracturing the samples under liquid nitrogen. The fractured surfaces were then sputter-coated with gold (Denton Vacuum, Desk V Sputter Coater).

Open-cell contents of porous PVDF samples were measured by a gas pycnometer (Quantachrome, Ultrapyc 1200e). Nitrogen was used to determine to total volume of the solid part and the closed-cell $\left(V_{t}\right)$ by employing Archimedes' principle of fluid displacement, and Boyle's Law of gas expansion. Then, the open-cell content $\left(O_{c}\right)$ can be determined by Equation (1).

$$
O_{c}=\frac{V_{g}-V_{t}}{V_{g}} \times 100 \%
$$

where $V_{g}$ is the geometric volume of the open-cell foam sample.

A simple contact-mode TENG, as illustrated in Figure 1, was constructed by using two pieces of cast acrylic plates as the substrates. Copper electrodes with conductive adhesive were adhered to the two plates. The positive and negative triboelectric layers were adhered to the two electrodes. Conducting wires were used to connect the two electrodes to an oscilloscope (Tektronix, TBS1052B-EDU) to measure the output voltage of the TENG during its operation. The measurement was obtained by averaging the output voltages generated by the mechanical vibration as a result of dropping the TENG's top plate, which was attached with a $195 \mathrm{~g}$ mass, onto its bottom plate for at least 25 times from a height of $3 \mathrm{~cm}$. All data points were obtained from the characterization of three samples and the error bars present the standard deviations of the data values. 


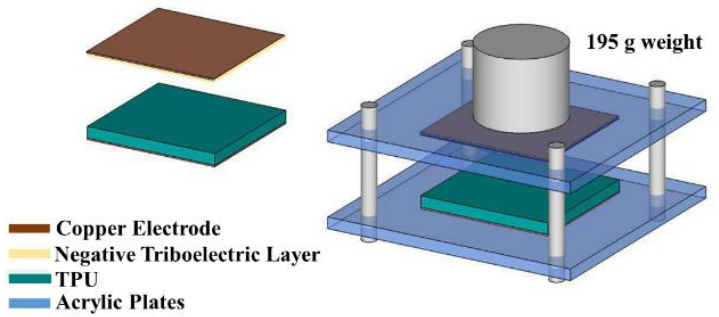

Figure 1. Schematic of Triboelectric Nanogenerator Setup

\section{RESULTS AND DISCUSSION}

The performance of TENG was predominantly depending on the degrees of triboelectrification in both the positive and the negative triboelectric layer. In this context, parametric studies were conducted to study the effects of polymer type, triboelectric layer's geometry, as well as foam morphology on the degrees of triboelectrification of these friction layers.

\section{A. Effect of Polymer Type on Triboelectricification}

The tendencies of materials to gain, lose, or hold electrons when contacting other materials depend on their chemical compositions. Literatures have reported different triboelectric series to organize materials according their ability to gain electrons. These data tables indicated that both PVDF and HDPE have higher tendencies than TPU to gain electrons. Therefore, when they are pairing up with TPU in a TENG, PVDF and HDPE will serve as the negative triboelectric layers while TPU will serve as the positive triboelectric layer. To compare their abilities to draw electrons away from TPU upon periodic contact and separation, solid PVDF and HDPE samples with the same contact area (i.e., $4 \mathrm{~cm}^{2}$ ) and varying thicknesses were used as the negative triboelectric layers of the TENG setup. Figure 2 illustrate the average peak voltages generated by TENG consisted of PVDF and HDPE as the negative triboelectric layers. It can be observed that, regardless of the layer's thickness, the peak voltages generated in the TENGs with PVDF negative triboelectric layers were $\sim 2$ times of those generated in the TENGs with HDPE negative triboelectric layers.

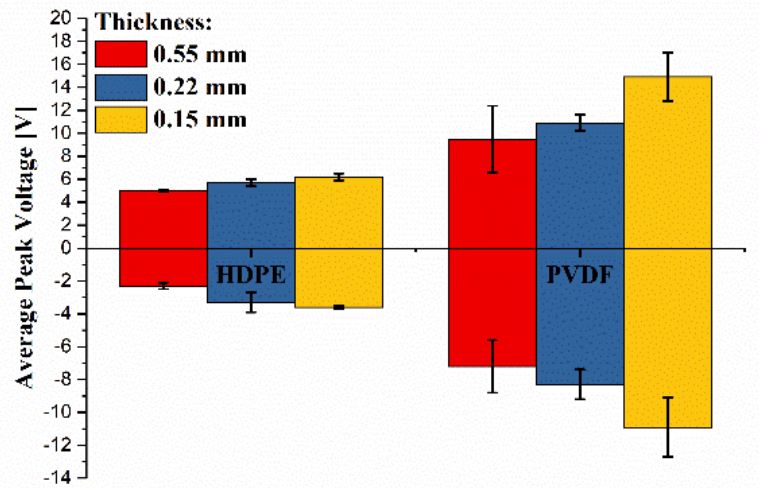

Film Type

Figure 2. Comparison of PVDF and HDPE's Performances on Triboelectrification for Contact Area of $4 \mathrm{~cm}^{2}$

\section{B. Effects of Sample Geometry on Triboelectricification}

The effects of triboelectric layer's thicknesses and contact area on the degree of triboelectrification were investigated by measuring the changes in vibration-induced voltage generated as these two geometric factors of the negative triboelectric layer (i.e., PVDF and HDPE) were varied. Figures 3 and 4 illustrate the effects of the thickness and contact area of the PVDF layer and the HDPE layer, respectively, on the peak voltages generated during periodic contact and separation of the TENG. Likewise, regardless of the thickness and the contact area, the uses of PVDF as the negative triboelectric layer consistently resulted in higher degrees of triboelectrification than the uses of HDPE.

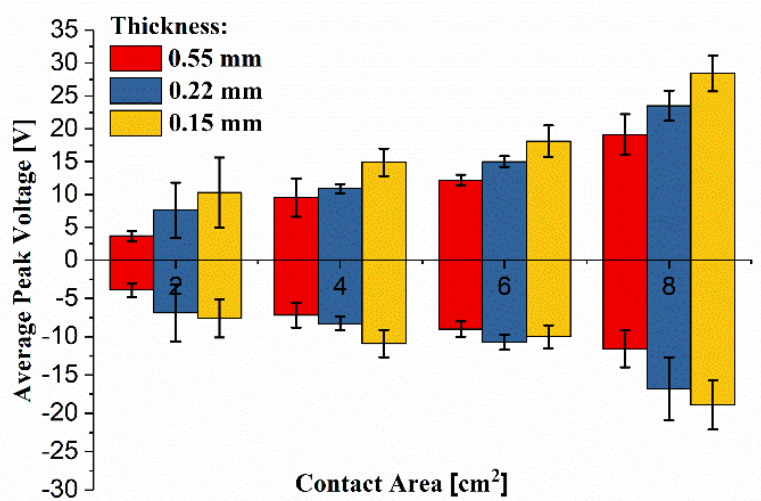

Figure 3. Effects of PVDF Layer's Geometry (i.e., Thickness and Contact Area) on Triboelectrification

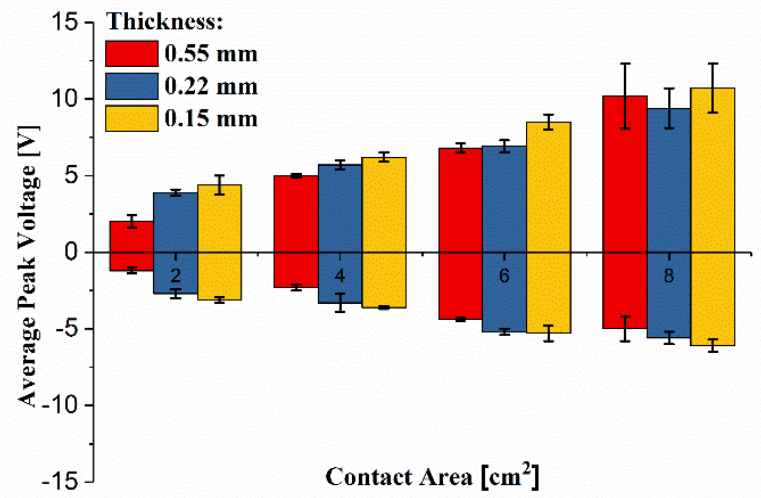

Figure 4. Effects of HDPE Layer's Geometry (i.e., Thickness and Contact Area) on Triboelectrification

For both PVDF and HDPE, it can also be observed that the peak output voltages of the TENG increased by increasing the triboelectric layer's contact area or decreasing the triboelectric layer's thickness. The effect of contact area on the degree of triboelectrification was intuitive. Increasing the contact area would result in the accumulation of more charges between the 
opposite triboelectric layers, leading to a higher degree of electrification during the periodic contact and seperation between the two layers. In constrast, decreasing the thickness of the PVDF or HDPE layer increased the peak output voltage. This could be attribute to the higher degree of electrostatic induction by the charged triboelectric layers and the electrodes. Experimental results confirmed the analytical model of the induced charge density (i.e., Equation (2)) derived by Wang et al. [7].

$$
\sigma^{\prime}=\frac{\sigma d_{3} \varepsilon_{r 1} \varepsilon_{r 2}}{d_{1} \varepsilon_{r 1}+d_{3} \varepsilon_{r 1} \varepsilon_{r 2}+d_{2} \varepsilon_{r 2}} \times 100 \%
$$

where $\sigma^{\prime}$ is the induced charge density; $\sigma$ is the triboelectric charge density $\varepsilon_{r 1}$ and $\varepsilon_{r 2}$ are the relative permittivity of the positive triboelectric layer and negative triboelectric layer, respectively; $d_{1}, d_{2}$, and $d_{3}$ are the thicknesses of the positive triboelectric layer, negative triboelectric layer, and the air gap, respectively.

\section{Effects of Open-Cell Morphology on Triboelectricification}

Figure 5 illustrates an SEM micrograph of a representative open-cell PVDF sample prepared by using $80 \mathrm{wt} \%$ of $\mathrm{NaCl}$ with particle size of $53 \mu \mathrm{m}-250 \mu \mathrm{m}$. The individual cells resembled the cubic shape of $\mathrm{NaCl}$ crystals. It can be observed that the cubic pores were uniformly distributed and interconnected throughout the PVDF matrices. Figure 6 revealed the effect of $\mathrm{NaCl}$ particle size (i.e., pore size after the particulate leaching process) on the open-cell contents of porous PVDF foams fabricated by using $80 \mathrm{wt} \% \mathrm{NaCl}$.

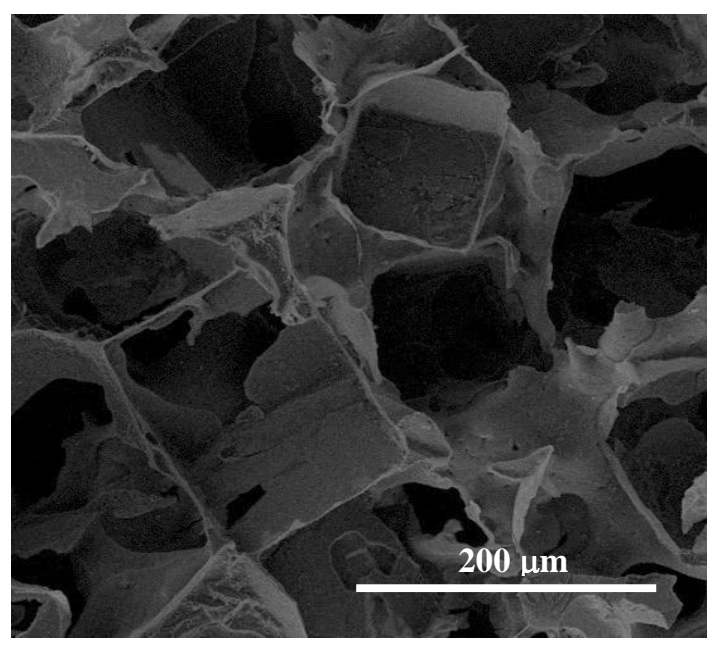

Figure 5. SEM Micrograph of Porous PVDF Negative Triboelectric Layer

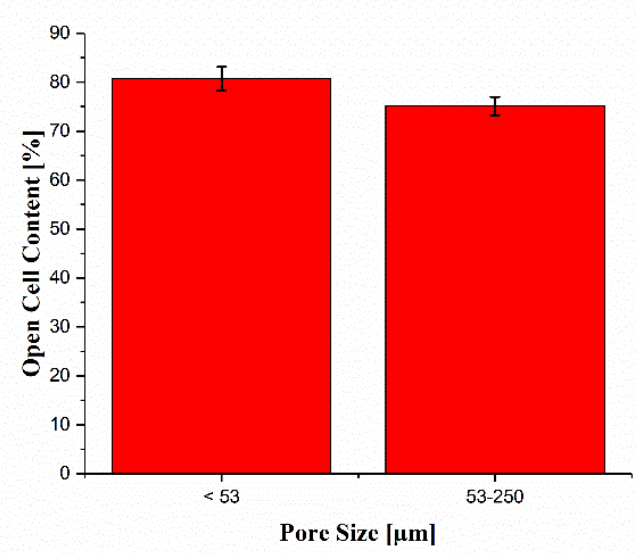

Figure 6. Effect of $\mathrm{NaCl}$ Particle Size on the Open-Cell Content of the Porous PVDF Layer

It can be observed that smaller $\mathrm{NaCl}$ particle sizes would yielded porous PVDF foams with higher open-cell contents. The negative correlation between these two parameters could be attributed to the higher packing efficiency of smaller particles. Figure 6 show that smaller $\mathrm{NaCl}$ particle size would increase the open-cell content of the fabricated PVDF foams after the complete leaching of $\mathrm{NaCl}$. The higher packing efficiency of smaller $\mathrm{NaCl}$ particles would promote the interconnectivity among the particles, increasing the opening among adjacent pores after the leaching process.

Figure 7 revealed that open-cell PVDF layer led to a higher degree of triboelectrification than solid PVDF layer. Furthermore, the promoted performance became more significant as the pore size decreased. It is believed that the presence of pores in the PVDF layer would enhance the degree of triboelectrification in three-fold. On the one hand, the ominpresence of pores throughout the PVDF layer significantly increased the surface area of the triboelectric layer. This would increase the accumulation of charges during the periodic contact and seperation of the opposite layers. On the other hand, this also reduced the local thickness of the triboelectric layer. It is speculated that this would enhance the electrostatic induction and thereby increased the peak voltage generated. Finally, the promoted conformabiity of the contact surfaces introduced by the PVDF foam structures would enhance adhension energy, which would then promote the triboelectric output [5]. 


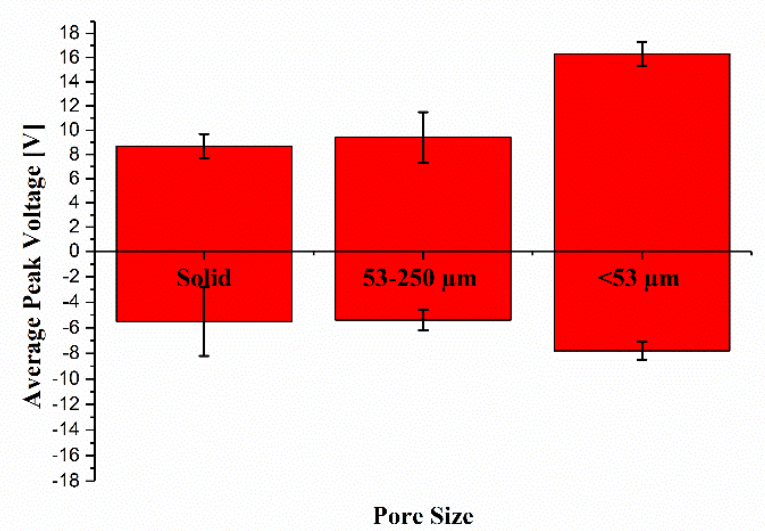

Figure 7. Effects of PVDF Layer's Morohology (Porosity) on Triboelectrification

\section{CONCLUSION}

The study has investigated the importance of polymer type, contact area, triboelectric layer thickness, and porosity on the triboelectric output voltage of TENG. Experimental results revealed that PVDF was a more efficient negative triboelectric material than HDPE, which increased the peak voltage by $\sim 2$ times. Furthermore, increasing the contact area and decreasing the triboelectric layer thickness positively influenced the degree of triboelectrification and/or electrostatic induction. Finally, particulate leaching, using $\mathrm{NaCl}$ as the leaching agent, could introduce open-cell structures within the PVDF layer. The presence of pores and the reduction in pore size were found to be effective strategies to promote the triboelectric output of TENG.

\section{ACKNOWLEDGMENT}

The authors are grateful about the financial support by the Natural Sciences and Engineering Research Council of Canada.

\section{REFERENCES}

[1] H. Peng, X. Sun, X. Fang, Polymer Materials for Energy and Electronic Applications, London, 2017, pp. 151-196.

[2] K. Y. Lee et al., "Hydrophobic sponge structure-based triboelectric nanogenerator," Adv. Mater., vol. 26, pp. 5037-5042, May 2014.

[3] Z. L. Wang, L. Lin, J. Chen, S. Niu, and Y. Zi, Triboelectric Nanogenerators, 1st ed., Cham, 2016, pp. 1-46.

[4] Z. L. Wang, "Triboelectric nanogenerators as new energy technology for self-powered systems and as active mechanical and chemical sensors," ACS Nano, vol. 7, pp. 9533-9557, November 2013.

[5] J. H. Lee, I. Yu, S. Hyun, J. K. Kim, and U. Jeong, "Remarkable increase in triboelectrification by enhancing the conformable contact and adhesion energy with a film-covered pillar structure," Nano Energy, vol. 34, pp. 233-241, January 2017.

[6] J. Chen et al., "Enhancing Performance of Triboelectric Nanogenerator by Filling High Dielectric Nanoparticles into Sponge PDMS Film," ACS Appl. Mater. Interfaces, vol. 8, pp. 736-744, 2016.

[7] J. A. Cross, "Electrostatics: Principles, Problems and Applications," Bristol, 1987, pp. 91-144. 